\title{
Preservation of electronic properties of double-decker complexes on metallic supports
}

Received 00th January 20xx, Accepted 00th January 20xx DOI: $10.1039 / x 0 x \times 00000 x$

www.rsc.org/

B. Cirera, ${ }^{a}$ J. Matarrubia, ${ }^{a}$ T. Kaposi, ${ }^{b}$ N. Giménez-Agulló, ${ }^{c}$ M. Paszkiewicz, ${ }^{b}$ F. Klappenberger, ${ }^{b}$ R. Otero, $^{\mathrm{a}, \mathrm{d}}$ J.M. Gallego, ${ }^{\mathrm{a}, \mathrm{e}}$ P. Ballester, ${ }^{\mathrm{c}, \mathrm{f}}$ J.V. Barth, ${ }^{\mathrm{b}}$ R. Miranda, ${ }^{\mathrm{a}, \mathrm{d}}$ J.R. Galán-Mascarós, ${ }^{*, c, f}$ W. Auwärter ${ }^{*}, \mathrm{~b}$ and D. Ecija*,a

\begin{abstract}
Single-molecule magnets based on lanthanide double-deckers are attracting great attention due to their unrivaled singleion anisotropy. In order to exploit their fascinating electronic and magnetic properties in devices for information storage or spin transport, studies on the preservation or variation of electronic and magnetic functionalities upon adsorption on surfaces are necessary. Here we introduce a comprehensive scanning tunneling microscopy (STM) and X-ray photoelectron spectroscopy (XPS) surface science study, complemented by density functional theory (DFT) simulations, of a recently synthesized single-molecule magnet based on porphyrazine deckers, conveniently equipped with ethyl moieties to make them soluble and sublimable. We show that the double-decker species are adsorbed intact on $\mathrm{Au}(111), \mathrm{Ag}(111)$ and $\mathrm{Cu}(111)$ in a flat-on fashion and self-assemble in hexagonal close-packed layers. Systematic multi- and monolayer XPS is performed on the surface-confined species, showing the preservation of the electronic properties of the ligands and the lanthanide center upon adsorption.
\end{abstract}

\section{Introduction}

In the last years increasing attention has been devoted to sandwich compounds embedding lanthanide ${ }^{1-22}$ or transition metal atoms, ${ }^{23-27}$ given their potential for information storage and spintronics. In particular, lanthanide phthalocyanine and porphyrin sandwich complexes (double-deckers) have emerged as a new paradigm in single molecular magnetism due to their unparalleled single-ion magnetic anisotropy (SMA), ${ }^{2}$ which is the origin for the appearance of single molecule magnet (SMM) behaviour. SMMs exhibit slow relaxation kinetics below a so-called blocking temperature, due to the presence of an activation energy that needs to be overcome for allowing magnetization reversal. ${ }^{3}$ This gives such single molecules many of the classical features of magnets (including hysteresis and memory effect), but at the single molecule level, which has brought tremendous attention on

a.IMDEA Nanoscience, 28049 Madrid, Spain. E-mail: david.ecija@imdea.org

b. Physik-Department E20, Technische Universität München, 85748 Garching Germany.E-mail:wau@tum.de

c. Institute of Chemical Research of Catalonia (ICIQ), The Barcelona Institute of Science and Technology, 43007, Tarragona, Spain.E-mail: jrgalan@iciq.es

d. Departamento Física de la Materia Condensada, Universidad Autónoma de Madrid, 28049 Madrid, Spain.

e. Instituto de Ciencia de Materiales de Madrid, CSIC, 28049 Madrid, Spain.

f. ICREA, Passeig Lluis Companys 23,08010, Barcelona, Spain.

Electronic Supplementary Information (ESI) available: STM images of the selfassembly of Dy(OETAP) ${ }_{2}$ on $\mathrm{Cu}(111)$. STM images of the orientational alignment of $\mathrm{Tb}(\mathrm{OETAP})_{2}$ species on $\mathrm{Ag}(111) .1 \mathrm{H}$ NMR spectra of Dy(OETAP $)_{2}$ as prepared and after sublimation. STM images and comparative height profiles of the selfassembly of OETAP and Dy $(\mathrm{OETAP})_{2}$ species on $\mathrm{Au}(111)$. STM images of the selfassembly of OETAP species on $\mathrm{Au}(111)$. See DOI: 10.1039/x0xx00000x this type of materials, aiming for future applications in information storage and spin transport.1, 4

An ideal single molecule magnet should be easy to process, preferentially soluble and sublimable, and at the same time preserve its electronic and magnetic properties upon deposition on distinct supports. Much effort has been invested in characterizing the properties of lanthanide double-deckers derivatives on surfaces, ${ }^{5-22}$ focusing not only on their relevance for molecular magnetism, but also on their potential for molecular machinery. ${ }^{19-21}$ However, a comprehensive understanding of the impact of the surface on their electronic and magnetic characteristics is still missing. Particularly astonishing is the shortage of $X$-ray photoelectron spectroscopy (XPS) studies, ${ }^{28-30}$ XPS being the most widespread technique to address spatially-averaged chemical and electronic properties of adsorbates. From very few studies based on X-ray magnetic circular dichroism, it is concluded that the impact of a metallic surface on the magnetic properties of a monolayer film of double-deckers can be dramatic, ${ }^{22}$ reducing the magnetic hysteresis loop when compared to a multilayer film ${ }^{31}$ or even closing it, $^{7}$ and thus, annihilating its potential use in devices. A promising strategy to protect the single-ion magnetic anisotropy of double-deckers is to equip them with decoupling terminal groups like ethyl moieties. As a result, upon flat-on adsorption and for specific adsorption conformations, the decoupling substituents could diminish the interaction of the lower macrocycle with the substrate, which could prevent the alteration of its electronic characteristics. It is convenient to emphasize that in order to preserve single-ion anisotropy for double-deckers incorporating Tb and Dy metals, 
which present an oblate f-electron charge cloud, it is required to concentrate the ligand electron density above and below the $x-y$ plane, and to preserve this distribution of ligand charge upon adsorption. Any modification of the electronic nature of the bottom ligand will most probably result in a diminishing of the single-ion magnetic anisotropy due to repulsive contacts between ligand and f-electron charge clouds. ${ }^{32}$

Here we introduce a systematic study of the adsorption, selfassembly and electronic properties of the recently synthesized family of $\left[\operatorname{Ln}(\mathrm{OETAP})_{2}\right]$ double-decker complexes $\left(\mathrm{Ln}=\mathrm{Dy}^{+3}\right.$, $\mathrm{Tb}^{+3}$; OETAP=octa(ethyl)tetraazaporphyrin). These $\operatorname{Ln}(\mathrm{OETAP})_{2}$ species are soluble and sublimable. Our combined scanning tunneling microscopy and XPS study reveal that the molecular species are deposited intact on well-defined pristine metal surfaces: $\mathrm{Dy}(\mathrm{OETAP})_{2}$ on $\mathrm{Au}(111)$ and $\mathrm{Cu}(111)$, and $\mathrm{Tb}(\mathrm{OETAP})_{2}$ on $\mathrm{Ag}(111)$. On all three coinage metal supports they selfassemble forming close-packed arrays. Importantly, systematic XPS experiments comparing monolayer versus multilayer coverage reveal an invariance of the electronic nature of the $\operatorname{Ln}(\mathrm{OETAP})_{2}$ species, i.e., first layer molecules are electronically almost identical to multilayer species, thus highlighting the preservation of their electronic characteristics.

\section{Experimental}

This work employs a complementary approach ${ }^{33}$ combining STM and XPS carried out in four different experimental ultrahigh vacuum (UHV) systems at a base pressure of $10^{-10}$ mbar: i) a SPECS variable-temperature STM set-up connected to a XPS chamber, ii) an OMICRON low-temperature STM, iii) a CreaTec low-temperature STM, and iv) a custom-design XPS set-up. $\mathrm{Au}(111), \mathrm{Ag}(111)$ and $\mathrm{Cu}(111)$ substrates were prepared by repeating cycles of annealing to $630 \mathrm{~K}, 650 \mathrm{~K}$ and $730 \mathrm{~K}$ respectively and $\mathrm{Ar}^{+}$ion sputtering. $\mathrm{Dy}(\mathrm{OETAP})_{2}$ and $\mathrm{Tb}(\mathrm{OETAP})_{2}$ molecules were evaporated by means of organic molecular beam epitaxy (OMBE) out of a quartz crucible heated up to 550K. All STM images were recorded with electrochemically etched W-tips in constant-current mode, applying the bias voltage to the sample. The XPS spectra were acquired by exposing the sample to $1486.6 \mathrm{eV}$ photons coming from the aluminum $\mathrm{K} \alpha$ transition and collecting the emitted electrons with a commercial hemispherical detector. Spectra have been fitted by Lorentzian functions directly on the acquired data; except for the nitrogen spectra on $\mathrm{Ag}(111)$, the dysprosium spectra on $\mathrm{Au}(111)$ and $\mathrm{Cu}(111)$, and the terbium spectra on $\mathrm{Ag}(111)$, for which the substrate background has been subtracted. Error bars correspond to the standard deviation of the experimental values.

DFT calculations were carried out by using the DMol3 package integrated in the Material Studio program of Accelrys Inc. ${ }^{34,} 35$ The exchange and correlation energies were calculated by using the generalized gradient approximation (GGA) of Perdew, Burke, and Ernzerhof. ${ }^{36}$ The valence electron functions were expanded into a set of numerical atomic orbitals by a double-numerical basis with polarization functions (DNP), (a polarization d-function on all non-hydrogen atoms and a polarization p-function on all hydrogen atoms). The cut-off radius was set at $\mathrm{Rc}=0.53 \mathrm{~nm}$. DFT semi-core pseudopotentials (DSPP), ${ }^{37}$ that include some degree of relativistic effects, were used for $\mathrm{Cu}, \mathrm{Y}$ and $\mathrm{Au}$. The Tkatchenko and Scheffler scheme ${ }^{38}$ for dispersion correction was also included. This method calculates the interatomic $C_{6}$ dispersion coefficients from rescaled atomic dispersion coefficients and polarizabilities, where the scaling factor is determined by the effective volume occupied by the atom in the molecular or solid environment. A $4 \times 4 \times 2$ Monkhorst-Pack k-point mesh ${ }^{39}$ has been used for all geometry optimization calculations. A four layers slab with a $\left(\begin{array}{ll}7 & 0 \\ 4 & 8\end{array}\right)$ unit cell (56 surface atoms per layer per unit cell) and a $\left(\begin{array}{cc}9 & 0 \\ 5 & 10\end{array}\right)$ unit cell for $\mathrm{Cu}$ (90 atoms per layer per unit cell) were used in the calculations. The substrate layers were kept fixed during geometry optimization, while all the atoms in the molecule were allowed to relax. Selfconsistency was considered achieved when the changes in total energy and force were smaller than $1 \times 10^{-6} \mathrm{Ha}$, and 0.001 $\mathrm{Ha} / \AA^{-1}$, respectively. Calculations were performed for $\mathrm{Y}(\mathrm{OETAP})_{2}$ and $\mathrm{Y}(\mathrm{TAP})_{2}$ molecular species.

\section{Results and discussion}

The single-molecule magnets $\operatorname{Dy}(\mathrm{OETAP})_{2}(\mathbf{1})$ and $\operatorname{Tb}(\mathrm{OETAP})_{2}$ (2), recently synthesized by us, ${ }^{40}$ are neutral double-decker compounds consisting of two octa(ethyl)tetraazaporphyrins coordinated by a lanthanide atom (Dy or Tb) in the center, featuring a square anti-prism geometry (cf. Figure 1a,b). These novel soluble molecular species exhibit high blocking temperatures exceeding 10 and $50 \mathrm{~K}$ in solution, respectively, which corresponds to an activation energy for magnetization reversal of $\approx 32 \mathrm{~K}($ Dy $3+)$ and $\approx 376 \mathrm{~K}(\mathrm{~Tb} 3+) .40$ As a result, they are promising candidates for devices exploiting molecular magnet functionalities.

$\mathrm{Dy}(\mathrm{OETAP})_{2}$ and $\mathrm{Tb}(\mathrm{OETAP})_{2}$ are ex-professo incorporating terminal ethyl moieties in order to enhance solubility, sublimation, and, importantly, to facilitate the decoupling of the double-decker molecular backbone upon adsorption on surfaces adopting a flat-on geometry.

In this work we study the adsorption, self-assembly and electronic properties of Dy(OETAP $)_{2}$ on $\mathrm{Cu}(111)$ and $\mathrm{Au}(111)$, and of $\mathrm{Tb}(\mathrm{OETAP})_{2}$ on $\mathrm{Ag}(111)$.

The deposition of a very low coverage of Dy(OETAP $)_{2}$ on $\mathrm{Cu}(111)$ and $\mathrm{Au}(111)$, and of $\mathrm{Tb}(\mathrm{OETAP})_{2}$ on $\mathrm{Ag}(111)$, results in step decoration by individual species, occasionally also found on the terraces. At negative bias, both species exhibit a central depression surrounded by eight lobular protrusions (cf. top panel of Figure 1c). By assuming a flat-on adsorption geometry, consistently reported for other double-decker compounds on metallic surfaces, and based on the molecular dimensions, each lobular protrusion can be assigned unambiguously to an ethyl terminal group. ${ }^{41}$ At positive bias, each two ethyls belonging to the same pyrrole moiety are imaged as an elongated protrusion (cf. bottom panel of Figure 1c). 


\section{PCCP}

\section{ARTICLE}
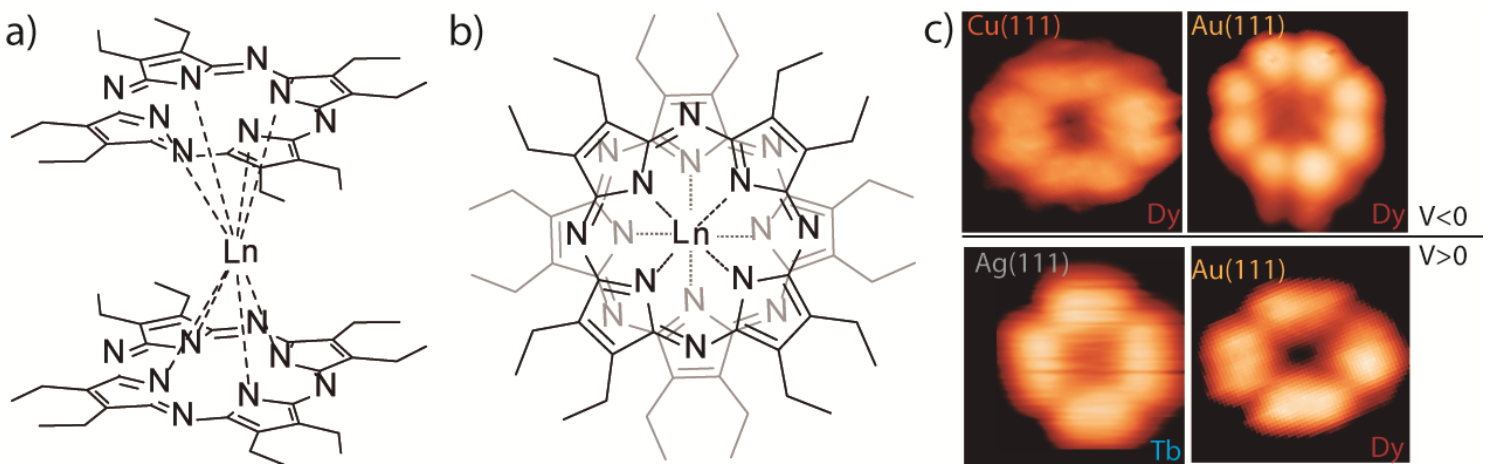

Figure 1 a) Chemical structure of the [Ln-(OETAP) $)_{2}$ illustrated in perspective. b) Top view of the molecular species with the top decker (gray) rotated 45 degrees with respect to the lower one (black) for clarity. c) STM images at positive and negative bias of individual Dy-(OETAP) ${ }_{2}$ (1) on Cu(111) and on Au(111), and Tb-(OETAP) 2 (2) species on Ag(111).

In order to model the adsorption of $\operatorname{Ln}(\mathrm{OETAP})_{2}$ species, simultaneously including a tractable computational description of the electronic valence band, which is under debate for DFT calculations of lanthanide elements involving f-orbitals, we studied the transition metal systems of $Y(O E T A P)_{2}$ and $Y(T A P)_{2}$ species on $\mathrm{Au}(111)$ and $\mathrm{Cu}(111)$. Yttrium exhibits the same oxidation state as $\mathrm{Dy} / \mathrm{Tb}$ in these compounds $(+3)$ and has a very similar ionic radii, being commonly used in solid state chemistry when a comparison of the structural or electronic properties with lanthanide-related compounds are necessary, 40 thus representing an ideal candidate to mimic structural conformations of lanthanide double-deckers on surfaces, as exemplified for the $\mathrm{TbPc}_{2}$ species on $\mathrm{Si}(100) .{ }^{29}$ Figure 2 illustrates that for the gas phase $\mathrm{Y}(\mathrm{OETAP})_{2}$ species the two $\pi$ backbones are just slightly bent, with the inner part closer to the $\mathrm{Y}$ atom, while the ethyl moieties are pointing outwards, making an angle of $110^{\circ}$ with the average backbone plane, due to the steric hindrance. Upon adsorption on $\mathrm{Ag}(111)$ this angle increases up to $127^{\circ}$.

a)

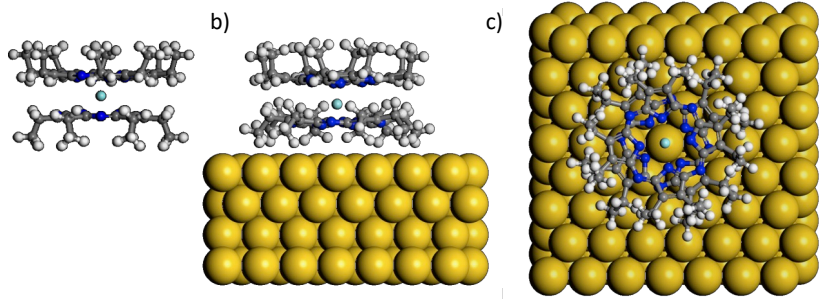

Figure 2. Results of DFT calculations showing a) a side view of an isolated $Y(O E T A P)_{2}$ molecule, b) side and c) top views of the geometry adsorption of $\mathrm{Y}(\mathrm{OETAP})_{2}$ species on $\mathrm{Au}(111) . \mathrm{H}, \mathrm{C}, \mathrm{N}, \mathrm{Y}$, and $\mathrm{Au}$ atoms are depicted in white, grey, blue, cyan and yellow, respectively.

Furthermore, the bending of the backbone planes is noticeably stronger, but now the inner part of the macrocycle is closer to the gold surface. The changes in the molecular conformation are due to a competition of: i) the van der Waals interactions between the $\pi$ backbone and the substrate, and ii) the steric hindrance between the bottom ethyl termini and the rest of the molecular compound. In the optimized adsorption geometry the distance between the $Y$ atom and the topmost surface plane is $5.3 \AA$. Also, the distance between the bottom macrocycle and the surface is $\sim 3.8 \AA$, which is $\sim 0.5 \AA$ larger than for related double-decker species without ethyl legs $\left(\mathrm{Y}(\mathrm{TAP})_{2}\right.$ compounds). In the case of $\mathrm{Cu}(111)$ this difference in adsorption height between the $\mathrm{Y}(\mathrm{OETAP})_{2}$ and the $\mathrm{Y}(\mathrm{TAP})_{2}$ species is even larger, amounting for $0.8 \AA$. The increased separations evidence a structural decoupling from the substrate due to the presence of the ethyl moieties, which also implies a larger electronic decoupling. Calculated charge density isosurfaces $\left(0.2 \mathrm{e} / \AA^{3}\right)$ for both $\mathrm{Y}(\mathrm{OETAP})_{2}$ and $\mathrm{Y}(\mathrm{TAP})_{2}$ species on $\mathrm{Cu}(111)$, show that the hybridization with the substrate electronic states is much smaller in the first case. By analogy, a similar structural and electronic decoupling is also expected for the dysprosium/terbium double-decker cases.

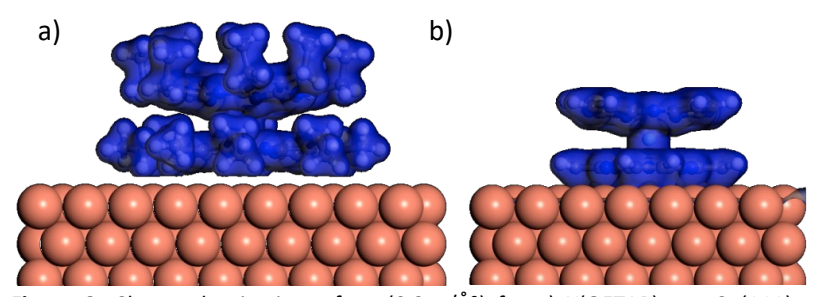

Figure 3. Charge density isosurface $\left(0.2\right.$ e/ $\left.\AA^{3}\right)$ for a) $\mathrm{Y}(\mathrm{OETAP})_{2}$ on $\mathrm{Cu}(111)$, and b) $\mathrm{Y}(\mathrm{TAP})_{2}$ on $\mathrm{Cu}(111)$.

The deposition of higher coverages of molecular species leads to the formation of analogous long-range ordered supramolecular architectures for the distinct double-decker 
molecular species and surfaces (cf. Figure 4 and Figure S1). Figure 4 shows a large-scale STM image, a high-resolution STM image, and a model of the close-packed assembly of (1) on $\mathrm{Au}(111)$ and of (2) on $\mathrm{Ag}(111)$. Molecular species are clearly distinguishable and identified as depressions (macrocycles) surrounded by eight or four protrusions (ethyl moieties at negative (cf. Figure $4 a, b$ ) and positive bias (cf. Figure 4c,d), respectively). The assembly is stabilized by van der Waals intermolecular interactions between adjacent ethyl substituents. The double-decker macrocycles span a quasihexagonal network that is characterized by the following experimental values: i) on $\mathrm{Au}(111)$, the lattice vector modules $|a|=14.2 \pm 0.5 \AA$ and $|b|=14.6 \pm 0.5 \AA$, enclosing an angle $\beta$ $=61 \pm 1^{\circ}$ (cf. Figure $4 \mathrm{~b}$ ); ii) on $\mathrm{Ag}(111)$ similar results are found with $|a|=14.0 \pm 0.5 \AA,|b|=14.2 \pm 0.5 \AA, \beta=66 \pm 1^{\circ}$ (cf. Figure 4d); and iii) on $\mathrm{Cu}(111)|a|=13.6 \pm 0.5 \AA,|b|=15.4 \pm 0.5 \AA$ and $\beta=63 \pm 1^{\circ}$ (cf. Figure S1). These supramolecular network parameters are very close to those encountered for $\mathrm{TbPc}_{2}$ on metals, which evolves as a result of the synergy of the ethyl moieties' flexibility and the axial rotation capability of the top macrocycle with respect to the bottom one that mediates to minimize steric repulsion. ${ }^{18}$
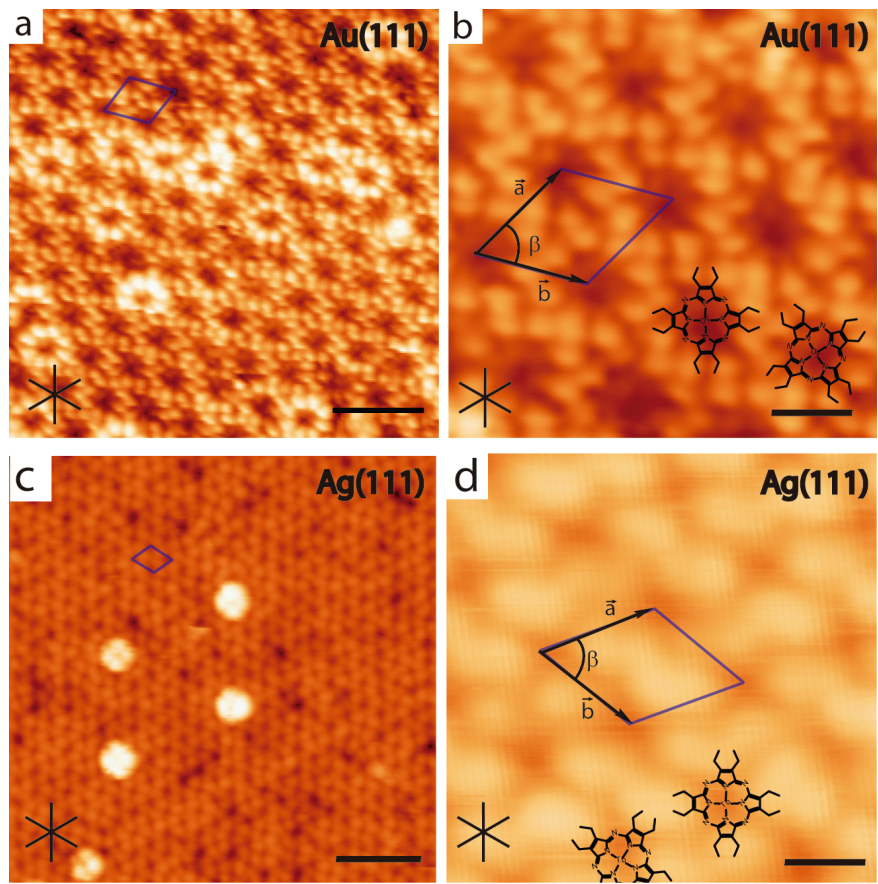

Figure. 4. Self-assembly of Ln-OETAP 2 species on close-packed noble metal surfaces. a) Large-scale STM image showing the self-assembly of (1) on $\mathrm{Au}(111)$ featuring a hexagonal network. The black star represents the high-symmetry directions of the $\mathrm{Au}(111)$ surface. Scale bar $=3 \mathrm{~nm}$. Tunneling parameters: $I_{t}=60 \mathrm{pA}$ and $V_{\text {bias }}=-2.3 \mathrm{~V}$. b) Zoomed-in STM image highlighting the quasi-hexagonal lattice described by the unit cell vectors, $\vec{a}$ and $\vec{b}$, and the angle $\beta$. Additionally, the superimposed model of (1) exhibits the correlation between the eight protrusions observed in the image and the eight ethyl legs of each top monomer. Scale bar $=1 \mathrm{~nm}$. Tunneling parameters: $I_{t}=60$ pA and $\mathrm{V}_{\text {bias }}=-2.3 \mathrm{~V}$. c) Large-scale STM image of the self-assembly of (2) on $\mathrm{Ag}(111)$ showing a hexagonal close-packed assembly similar to the one of (1). Scale bar $=6 \mathrm{~nm}$. Tunneling parameters: $\mathrm{l}_{\mathrm{t}}=100 \mathrm{pA}$ and $\mathrm{V}_{\text {bias }}=1.5 \mathrm{~V}$. d) High-resolution STM image showing the unit cell vectors, which are analogous to those found for the adsorption and self-assembly of $(\mathbf{1})$ on $\mathrm{Cu}(111)$ and on $\mathrm{Au}(111)$. Scale bar $=1 \mathrm{~nm}$. Tunneling parameters: $\mathrm{I}_{\mathrm{t}}=100 \mathrm{pA}$ and $\mathrm{V}_{\text {bias }}=1.5 \mathrm{~V}$.
Importantly, two alternating molecular orientations with respect to the substrate are distinguished along the network directions that are related by a $30^{\circ}$ rotation. Each of these oriented species align one of the symmetry axis of the top macrocycle with one of the high symmetry directions (cf. Figure S2). Occasionally observed second layer double-decker entities (bright species in Figure 4c) are easily removable by scanning with normal imaging conditions.

In order to gain insights on the integrity of the deposited species, we focused on the Dy $(\text { OETAP })_{2}$ species and performed two distinct experiments. First, deposition under vacuum conditions on a cold anode and subsequent NMR showed that intact $\left.\left[\mathrm{Dy}(\mathrm{OETAP})_{2}\right)\right]$ were sublimated from the crucible (cf. Figure S3). Second, to discard on-surface molecular cracking due to the interaction with the substrate, we sublimated freebase OETAP (3) molecules on Au(111). In agreement to recently published results, ${ }^{41}$ OETAP self-assembles into a rhombic superlattice (Figure S4 and Figure S5). Importantly, a comparison of the respective apparent heights at $\mathrm{V}_{\text {bias }}=-1.5 \mathrm{~V}$ reveals a higher value for the double-decker entities (4.4 vs. 1.4 ̊), which qualitatively supports our argumentation. A third fact in favor of intact adsorption and self-assembly of the single molecule magnets is the lack of statistically relevant broken species on the surfaces after the deposition of $\operatorname{Dy}(\mathrm{OETAP})_{2}$ or $\mathrm{Tb}(\mathrm{OETAP})_{2}$. Importantly, this holds even on the more reactive $\mathrm{Cu}(111)$ substrate, in contrast to other doubledecker derivatives. ${ }^{5}, 21,28$ Accordingly, our findings highlight the potential of $\mathrm{Dy}(\mathrm{OETAP})_{2}$ (and analogously of $\mathrm{Tb}(\mathrm{OETAP})_{2}$ ) for applications demanding thermal sublimation.

After comprehensively describing the geometrical properties of the supramolecular lattices, we now present the XPS measurements investigating the chemical and electronic properties of (1) and (2) upon adsorption. Figure 5 shows the spectra for the C1s, N1s and Dy $3 d_{5}$ core levels for (1) at monolayer and multilayer regime on $\mathrm{Au}(111)$ and $\mathrm{Cu}(111)$.

First, we focus on the monolayers and compare the influence of the different surfaces. As illustrated in the top panel of Figure $5 \mathrm{a}$, the C1s spectra (top left panel), the N1s peak (top middle panel), and the Dy $3 d_{5}$ spectra (top right panel) show negligible energy shifts and minute shape variations between the samples prepared on different substrates. The invariance against the varying reactivity of the two surfaces indicates minor interactions between the double-decker core and the metallic environment.

Next, we studied multilayer depositions on the same surfaces to gain insights to molecular species not interacting with the substrate. The respective $\mathrm{C} 1 \mathrm{~s}$ and the $\mathrm{N} 1 \mathrm{~s}$ core levels are only shifted 0.1 and $0.2 \mathrm{eV}$ (cf. bottom panels in Figure 5a and b). In the monolayer regime, the flat-on adsorption of the doubledecker species implies that one tetra-azaporphyrin ligand is in direct contact with the surface, which is associated with the origin of this very small binding energy shift. Notably, the dysprosium spectra are barely changed, indicating that the original electronic and chemical state of the compound and its metal center are protected. Specifically, the $3+$ oxidation state of the rare earth center seems to be preserved. 

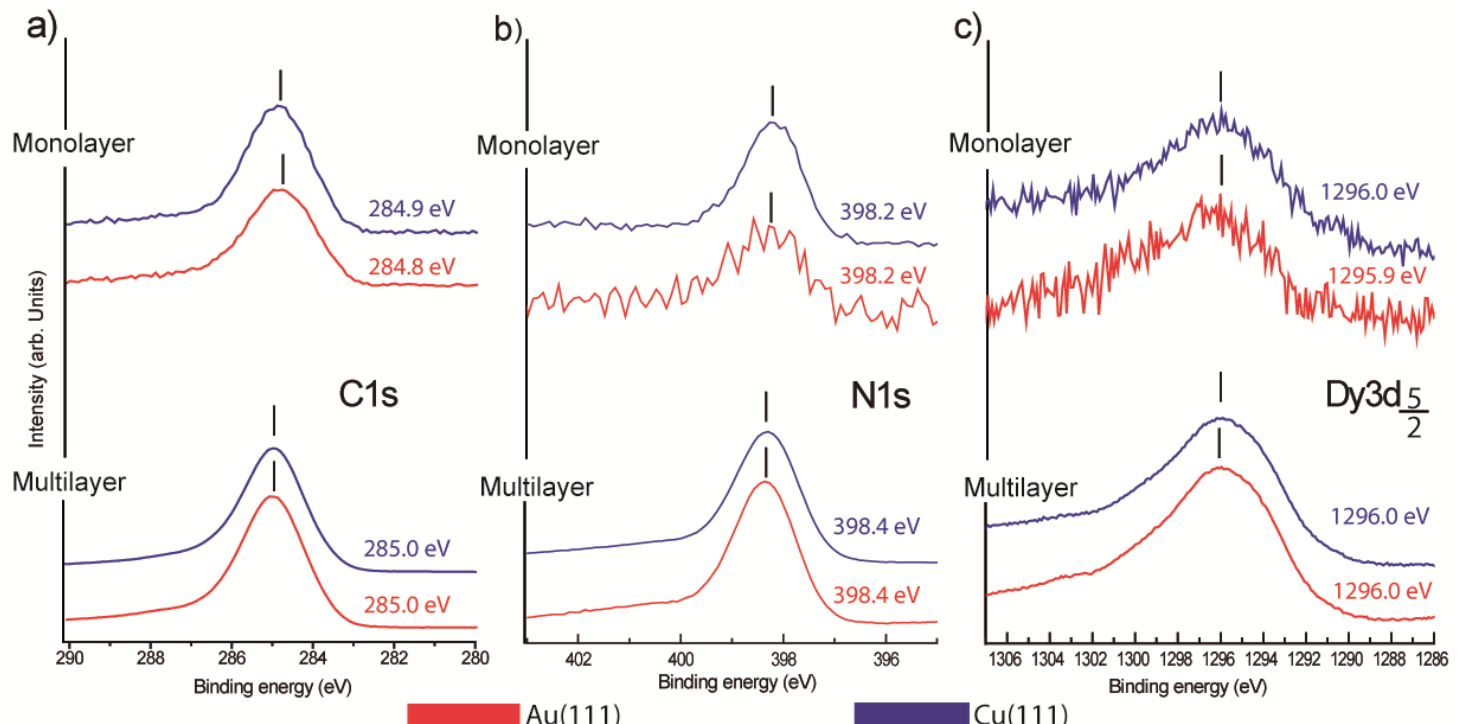

Figure 5. XPS spectra of the deposition of (1) for monolayer and multilayer regimes on $\mathrm{Au}(111)$ and on $\mathrm{Cu}(111)$, respectively, indicating a minute and negligible influence of the distinct surfaces. Red and blue spectra represent samples grown on $\mathrm{Au}(111)$ and $\mathrm{Cu}(111)$, respectively. a) $\mathrm{C} 1 \mathrm{~s}$ core level spectra for the monolayer and the multilayer regime. b) Spectra for the N1s level featuring an energy shift of $0.2 \mathrm{eV}$ to higher energies in the multilayer sample. c) Dy $3 d_{\frac{5}{2}}$ spectra showing minute differences between the multilayer and the monolayer.

Furthermore, a similar comparison of multilayer vs. monolayer regime for the Tb-species (2) on $\mathrm{Ag}(111)$ is depicted in Figure 6. Again the $\mathrm{C} 1 \mathrm{~s}$ and $\mathrm{N} 1 \mathrm{~s}$ signatures remain largely unaffected by the substrate. The $\mathrm{Tb}_{3} \mathrm{~d}_{3 / 2}$ peak appears to be centered at the same binding energy as in the multilayer, even though the minute amount of $\mathrm{Tb}$ in the monolayer sample obstructs a clear determination of the exact shape. In view of the prominent shifts of the Fe and Co peaks discussed before for single-decker systems, our findings indicate that the effect of the substrate on the electronic properties of $\mathrm{Tb}(\mathrm{OETAP})_{2}$ is similarly negligible as for the Dy- double-decker species.
Based on our results, we argue that neither the ligand's nor the metal center's chemical and electronic properties are importantly modified upon adsorption on coinage metal surfaces, which is a prerequisite to preserve single-ion magnetic anisotropy. Since SMA can be quenched by minor distortions of the molecular structure, such as azimuthal rotational angle between the ligands and symmetry of the bonding around lanthanide ions, further studies on magnetic properties of surface-confined $\mathrm{Dy}(\mathrm{OETAP})_{2}$ and $\mathrm{Tb}(\mathrm{OETAP})_{2}$ species are envisioned to elucidate the preservation (or minor variation) of the single-ion magnetic anisotropy.
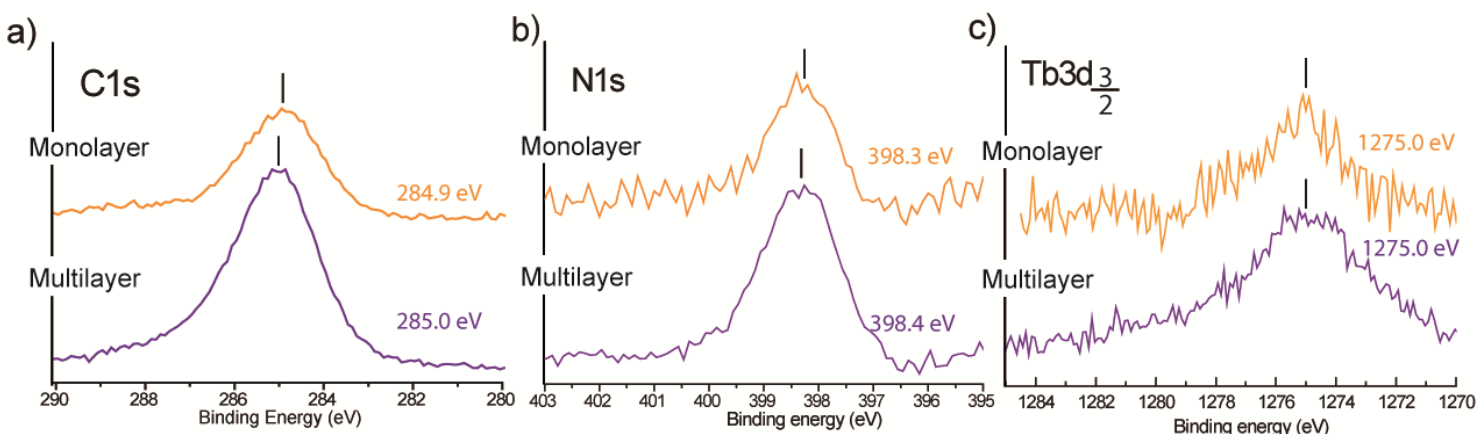

Figure 6. a) XPS spectra of the C1s core level for (2) deposited on $\mathrm{Ag}(111)$ in the monolayer (orange) and multilayer (violet) regime, exhibiting a minor shift of $0.1 \mathrm{eV}$ in agreement with species (1). b) Similar results for the N1s core level. c) Tb $3 d_{\frac{3}{2}}$ monolayer and multilayer spectra, demonstrating the decoupling of Tb from the surface. 
6. F. Branzoli, P. Carretta, M. Filibian, G. Zoppellaro, M. J. Graf, J. R. Galan-Mascaros, O. Fuhr, S. Brink and M. Ruben, J. Am. Chem. Soc., 2009, 131, 7934-7934.

\section{Conclusions}

We present a comprehensive STM, XPS and DFT study of the adsorption, self-assembly, chemical and electronic properties of Dy $(\mathrm{OETAP})_{2}$ and $\mathrm{Tb}(\mathrm{OETAP})_{2}$ on coinage metals. The doubledecker species were conveniently functionalized with ethyl moieties to be soluble and sublimable. Importantly, the groups also help to decouple the double-decker core entities from the surface. We prove the deposition of intact molecular species on $\mathrm{Au}(111), \mathrm{Cu}(111)$, and $\mathrm{Ag}(111)$ adopting a flat-on geometry and show their assembly in hexagonal close-packed networks. Systematic XPS studies reveal a negligible influence of the surface on the electronic properties of the ligands and the lanthanide center, which behavior is prerequisite for the protection of their single-ion magnetic anisotropy. We aim to fully explore these prospects in the field of molecular magnetism and spintronics in future studies.

\section{Acknowledgements}

We acknowledge financial support of the EU (projects ERC Advanced Grant MolArt (no. 247299), ERC Consolidator Grant Nanosurfs (no. 615233), ERC Starting Grant CHEMCOMP (no. 279313), EC FP7-PEOPLE-2011-COFUND AMAROUT II program), the Spanish Ministerio de Economía y Competitividad (MINECO) (RYC-2012-11133, FIS 2013-40667-P, FIS 2015-67287-P and Severo Ochoa Excellence Accreditation 2014-2018 SEV-2013-0319), the Comunidad de Madrid (project MAD2D), Generalitat de Catalunya (2014-SGR-797), the IMDEA Foundation, the ICIQ Foundation, the German Research Foundation (DFG) via KL 2294/3-1, and the Munich-Center for Advanced Photonics (MAP). WA acknowledges funding by the DFG via a Heisenberg professorship.

\section{References}

1. D. N. Woodruff, R. E. P. Winpenny and R. A. Layfield, Chem. Rev., 2013, 113, 5110-5148.

2. N. Ishikawa, M. Sugita, T. Ishikawa, S. Koshihara and Y. Kaizu, J. Am. Chem. Soc., 2003, 125, 8694-8695.

3. N. Ishikawa, M. Sugita and W. Wernsdorfer, Angew. Chem. Int. Ed., 2005, 44, 2931-2935.

4. R. Sessoli and A. K. Powell, Coord. Chem. Rev., 2009, 253, 2328-2341.

5. L. Vitali, S. Fabris, A. M. Conte, S. Brink, M. Ruben, S. Baroni and K. Kern, Nano Lett., 2008, 8, 3364-3368.
7. S. Stepanow, J. Honolka, P. Gambardella, L. Vitali, N. Abdurakhmanova, T. Tseng, S. Rauschenbach, S. L. Tait, V. Sessi, S. Klyatskaya, M. Ruben and K. Kern, J. Am. Chem. Soc., 2010, 132, 11900-11901.

8. A. Lodi Rizzini, C. Krull, T. Balashov, J. J. Kavich, A. Mugarza, P. S. Miedema, P. K. Thakur, V. Sessi, S. Klyatskaya, M. Ruben, S. Stepanow and P. Gambardella, Phys. Rev. Lett., 2011, 107.

9. M. Urdampilleta, S. Klyatskaya, J. P. Cleuziou, M. Ruben and W. Wernsdorfer, Nat. Mater., 2011, 10, 502-506.

10. Y.-S. Fu, J. Schwöbel, S.-W. Hla, A. Dilullo, G. Hoffmann, S. Klyatskaya, M. Ruben and R. Wiesendanger, Nano Lett., 2012, 12, 3931-3935.

11. A. Lodi Rizzini, C. Krull, T. Balashov, A. Mugarza, C. Nistor, F. Yakhou, V. Sessi, S. Klyatskaya, M. Ruben, S. Stepanow and P. Gambardella, Nano Lett., 2012, 12, 5703-5707.

12. J. Schwöbel, Y. Fu, J. Brede, A. Dilullo, G. Hoffmann, S. Klyatskaya, M. Ruben and R. Wiesendanger, Nat. Commun., 2012, 3, 953.

13. M. Ganzhorn, S. Klyatskaya, M. Ruben and W. Wernsdorfer, Nat. Nanotech., 2013, 8, 165-169.

14. K. Katoh, Y. Yoshida, M. Yamashita, H. Miyasaka, B. K. Breedlove, T. Kajiwara, S. Takaishi, N. Ishikawa, H. Isshiki, Y. F. Zhang, T. Komeda, M. Yamagishi and J. Takeya, J. Am. Chem. Soc., 2009, 131, 9967-9976.

15. T. Komeda, H. Isshiki, J. Liu, Y.-F. Zhang, N. Lorente, K. Katoh, B. K. Breedlove and M. Yamashita, Nat. Commun., 2011, 2, 217.

16. R. Robles, N. Lorente, H. Isshiki, J. Liu, K. Katoh, B. K. Breedlove, M. Yamashita and T. Komeda, Nano Lett., 2012, 12, 3609-3612.

17. T. Komeda, H. Isshiki, J. Liu, K. Katoh, M. Shirakata, B. K. Breedlove and M. Yamashita, ACS Nano, 2013, 7, 10921099.

18. T. Komeda, K. Katoh and M. Yamashita, Prog. Surf. Sci., 2014, 89, 127-160.

19. K. Miyake, M. Fukuta, M. Asakawa, Y. Hori, T. Ikeda and T. Shimizu, J. Am. Chem. Soc., 2009, 131, 17808-17813.

20. J. Otsuki, Y. Komatsu, D. Kobayashi, M. Asakawa and K. Miyake, J. Am. Chem. Soc., 2010, 132, 6870-6871.

21. D. Écija, W. Auwärter, S. Vijayaraghavan, K. Seufert, F. Bischoff, K. Tashiro and J. V. Barth, Angew. Chem. Int. Ed., 2011, 50, 3872-3877.

22. C. Wäckerlin, F. Donati, A. Singha, R. Baltic, S. Rusponi, K. Diller, F. Patthey, M. Pivetta, Y. Lan, S. Klyatskaya, M. Ruben, H. Brune and J. Dreiser, Adv. Mater., 2016, 28, 5195-5199.

23. R. A. Layfield, Organometallics, 2014, 33, 1084-1099.

24. B. W. Heinrich, L. Limot, M. V. Rastei, C. lacovita, J. P. Bucher, D. M. Djimbi, C. Massobrio and M. Boero, Phys. Rev. Lett., 2011, 107, 216801. 


\section{Journal Name}

25. M. Ormaza, P. Abufager, N. Bachellier, R. Robles, M. Verot, T. Le Bahers, M.-L. Bocquet, N. Lorente and L. Limot, J. Phys. Chem. Lett., 2015, 6, 395-400.

26. B. Özdamar, C. Massobrio and M. Boero, J. Phys. Chem. C, 2016, 120, 13825-13830.

27. M. Ormaza, R. Robles, N. Bachellier, P. Abufager, N. Lorente and L. Limot, Nano Lett., 2016, 16, 588-593.

28. M. Toader, M. Knupfer, D. R. T. Zahn and M. Hietschold, J. Am. Chem. Soc., 2011, 133, 5538-5544.

29. M. Mannini, F. Bertani, C. Tudisco, L. Malavolti, L. Poggini, K. Misztal, D. Menozzi, A. Motta, E. Otero, P. Ohresser, P. Sainctavit, G. G. Condorelli, E. Dalcanale and R. Sessoli, Nat. Commun., 2014, 5.

30. I. Bidermane, J. Lüder, S. Ahmadi, C. Grazioli, M. Bouvet, B. Brena, N. Mårtensson, C. Puglia and N. Witkowski, J. Phys. Chem. C, 2016, 120, 14270-14276.

31. L. Margheriti, D. Chiappe, M. Mannini, P. Car, P. Sainctavit, M. A. Arrio, F. B. de Mongeot, J. C. Cezar, F. M. Piras, A. Magnani, E. Otero, A. Caneschi and R. Sessoli, Adv. Mater., 2010, 22, 5488-5493.

32. J. D. Rinehart and J. R. Long, Chem. Sci., 2011, 2, 2078.

33. F. Klappenberger, Prog. Surf. Sci., 2014, 89, 1-55.

34. B. Delley, J. Chem. Phys., 1990, 92, 508.

35. B. Delley, J. Chem. Phys., 2000, 113, 7756.

36. J. P. Perdew, W. Burke and M. Ernzerhof, Phys. Rev. Lett., 1996, 77, 3965.

37. B. Delley, Phys. Rev. B, 2002, 66, 155125.

38. E. R. McNellis, J. Meyer and K. Reuter, Phys. Rev. B, 2009, 80, 205414.

39. H. J. Monkhorst and J. D. Pack, Phys. Rev. B, 1976, 13, 5188.

40. N. Giménez-Agulló, C. S. de Pipaón, L. Adriaenssens, M. Filibian, M. Martínez-Belmonte, E. C. Escudero-Adán, P. Carretta, P. Ballester and J. R. Galán-Mascarós, Chem. Eur. J., 2014, 20, 12817-12825.

41. B. Cirera, N. Gimenez-Agullo, J. Bjork, F. Martinez-Pena, A. Martin-Jimenez, J. Rodriguez-Fernandez, A. M. Pizarro, R. Otero, J. M. Gallego, P. Ballester, J. R. Galan-Mascaros and D. Ecija, Nat. Commun., 2016, 7. 